\title{
100 Years of Cinema: Looking Ahead
}

\author{
By The Editor
}

Fall 1995 Issue of KINEMA

IN THIS CENTENNARY YEAR OF FILM, much has been said about cinema's past; now may be time to see what lies ahead in view of the rapidly approaching new century and millennium. We do not know which form the film medium is going to assume a hundred years from now and, indeed, if it is going to exist at all.

It is, however, not my intention to look this far ahead. Let us glance instead at the very near future of this journal, and see the form it will take in the time to come. The first issue of KINEMA was published only in 1993. The word "Netscape" was still unknown then. Today, the name of this browser software is a household word and millions of people around the globe, young and old, put on its wings and journey out to explore the magic Web. Many of them enter the Internet to read the flood of books, journals and magazines available there -- their numbers are growing exponentially.

It is now clear that there is little reason why KINEMA should remain outside of this remarkable cultural transformation. The properties of the electronic journal are in fact well-suited to the philosophical concept of this periodical: to be a truly international journal which addresses the film and audiovisual media as a global phenomenon. The Internet can, among other things, virtually eliminate most of the physical and economical barriers of KINEMA's delivery to its readers anywhere in the world, even in the most isolated places on the globe, thus effectively multiplying its reach and providing an efficient feedback at the same time.

The form of electronic publication, however, offers much more than that. The style used by authors in their scholarly articles will be significantly influenced by the potential of the hypertext which virtually removes the physical limits of the conventional academic text's reference apparatus. Any name, term, or word can be linked to another, if the author desires. Such references may include conventional text sources, images, sounds, and moving pictures. Articles published in electronic form can be easily updated or revised, reflecting new information about the given subject. This type of text will eventually lead to a new kind of integrated, multi-layered reading experience. Whatever the physical limitations of electronic publishing today, they will diminish as time goes by. Much more could be said about the potential of electronic text and hypertext but this would extend far beyond the scope of a simple editor's note.

Earlier this year, the KINEMA site was added to Internet's World Wide Web. As of now, it includes the Spring 1993 issue in full and selected articles from the more recent issues. Since the Spring 1994 and 1995 issues of KINEMA are both out of print, the Internet site will help us to make them available electronically to all readers.

The Internet version of KINEMA is not meant to replace the printed journal which will remain available to those who wish to obtain it. For many, the act of reading from a printed text provides aesthetic and tactile pleasure; others would like to keep their journal in a more tangible form than on a computer diskette. Moreover, not everybody has access to a computer, and a number of us are simply not interested in getting one. The two KINEMAs -- the physical printout and the electronic one -- will coexist in our multifaceted world. 